\title{
Reverse Order Law for the Core Inverse in Rings
}

\author{
Honglin Zou*, Jianlong Chen†, Pedro Patrício ${ }^{\ddagger}$ \\ ${ }^{\star}$ College of Mathematics, Yangzhou University, Yangzhou 225002, China \\ *School of Mathematics and Statistics, Hubei Normal University, \\ Huangshi 435002, China \\ †School of Mathematics, Southeast University, Nanjing 210096, China \\ ${ }^{\ddagger}$ CMAT-Centro de Matemática and Departamento de Matemática e Aplicações, \\ Universidade do Minho, Braga 4710-057, Portugal
}

\begin{abstract}
In this paper, necessary and sufficient conditions of the one-sided reverse order law $(a b)^{\oplus}=b^{\oplus} a^{\oplus}$, the two-sided reverse order law $(a b)^{\oplus}=b^{\oplus} a^{\oplus}$ and $(b a)^{\oplus}=$ $a^{\boxplus} b^{\oplus}$ for the core inverse are given in rings with involution. In addition, the mixed-type reverse order laws, such as $(a b)^{\#}=b^{\oplus}\left(a b b^{\boxplus}\right)^{\boxplus}, a^{\boxplus}=b(a b)^{\#}$ and $(a b)^{\#}=b^{\oplus} a^{\boxplus}$, are also considered.
\end{abstract}

Keywords: core inverse; group inverse; reverse order law

AMS Subject Classifications: 15A09; 16W10; 16 B99

\section{Introduction}

Let $\mathbb{C}_{n \times n}$ denote the set of all $n \times n$ complex matrices. It is well known that $(A B)^{-1}=$ $B^{-1} A^{-1}$, where $A, B \in \mathbb{C}_{n \times n}$ are invertible. The previous equality is called the reverse order law for the ordinary inverse. In general, the equality doesn't hold when the ordinary inverse is replaced by the generalized inverse. In 1966, Greville [13] first gave a necessary and sufficient condition of the reverse order law $(A B)^{\dagger}=B^{\dagger} A^{\dagger}$ for the Moore-Penrose inverse. Since then, many authors studied the reverse order law for various classes of generalized inverses in the setting of complex matrices, operators, and elements of rings with involution. For example, Deng [10] investigated some necessary and sufficient conditions of the reverse order law $(a b)^{\#}=b^{\#} a^{\#}$ for the group inverse of linear bounded operators on Hilbert spaces. In [23], Mosić and Djordjević extended the results of [10] to the ring case, giving some new conditions and providing simpler and more transparent proofs to already existing conditions. Recently, Mary [19] provided equivalent conditions for the two-sided reverse order law $(a b)^{\#}=b^{\#} a^{\#}$ and $(b a)^{\#}=a^{\#} b^{\#}$ for the group inverse in semigroups and rings. In [20, 22, 24], Mosić

\footnotetext{
${ }^{*}$ Corresponding author. E-mail: honglinzou@163.com

${ }^{\dagger}$ E-mail: jlchen@seu.edu.cn

${ }^{\ddagger}$ E-mail: pedro@math.uminho.pt
} 
et al. considered the mixed-type reverse order laws in rings, such as $(a b)^{\dagger}=b^{\dagger}\left(a^{\dagger} a b b^{\dagger}\right)^{\dagger} a^{\dagger}$, $(a b)^{\#}=b^{\dagger}\left(a^{\dagger} a b b^{\dagger}\right)^{\dagger} a^{\dagger},(a b)^{\#}=\left(a^{\dagger} a b\right)^{\dagger} a^{\dagger}$, and $(a b)^{\#}=b^{\dagger} a^{\dagger}$. More results on the reverse order law for the generalized inverse can be found in $[3,7-9,11,16-18,21,25,29]$.

The following problem on the reverse order law for the core inverse was proposed by Baksalary and Trenkler [2]:

$$
\text { If } A^{\oplus}, B^{\oplus} \text {, and }(A B)^{\oplus} \text { exist, does it follow that }(A B)^{\oplus}=B^{\oplus} A^{\oplus} \text { ? }
$$

This problem attracted researchers' attention. Later, Cohen, Herman and Jayaraman [6] gave several counterexamples for the problem. In [30], Wang and Liu obtained equivalent conditions of the reverse order $(A B)^{\oplus}=B^{\oplus} A^{\oplus}$ by the ranks of matrices. Next, we will continue to consider the reverse law for the core inverse in rings with involution.

The article is motivated by the papers $[19,22,30]$. We present some equivalent conditions for the one-sided reverse order law $(a b)^{\oplus}=b^{\oplus} a^{\oplus}$, the two-sided reverse order law $(a b)^{\oplus}=$ $b^{\oplus} a^{\oplus}$ and $(b a)^{\oplus}=a^{\oplus} b^{\oplus}$ for the core inverse in rings with involution. We also study the mixed-type reverse order laws, such as $(a b)^{\#}=b^{\oplus}\left(a b b^{\oplus}\right)^{\boxplus}, a^{\boxplus}=b(a b)^{\#}$ and $(a b)^{\#}=b^{\oplus} a^{\boxplus}$.

\section{Preliminaries}

Throughout this paper, $R$ denotes a unital $*$-ring, that is, a ring with unity 1 and an involution $a \mapsto a^{*}$ satisfying $\left(a^{*}\right)^{*}=a,(a+b)^{*}=a^{*}+b^{*}$ and $(a b)^{*}=b^{*} a^{*}$ for all $a, b \in R$.

For the readers' convenience, we first recall the definitions of some generalized inverses. An element $a \in R$ is said to be Moore-Penrose invertible with respect to the involution $*$ if the following equations

$$
\text { (1) } a x a=a, \quad(2) x a x=x, \quad(3)(a x)^{*}=a x, \quad(4)(x a)^{*}=x a
$$

have a common solution [26]. Such solution is unique if it exists, and is denoted by $a^{\dagger}$.

The Drazin inverse [12] of $a \in R$ is the element $x \in R$ which satisfies

$$
\left(1^{k}\right) a^{k}=a^{k+1} x, \quad(2) x a x=x, \quad(5) a x=x a, \text { for some } k \geq 1 .
$$

The element $x$ above is unique if it exists and is denoted by $a^{D}$. The smallest such $k$ is called the index of $a$, and denoted by ind $(a)$. In particular, when ind $(a)=1$, the Drazin inverse $a^{D}$ is called the group inverse of $a$ and it is denoted by $a^{\#}$.

Baksalary and Trenkler [1] introduced the core inverse for complex matrices. Let $A \in$ $\mathbb{C}_{n \times n}$. A matrix $A^{\oplus} \in \mathbb{C}_{n \times n}$ is called core inverse of $\mathrm{A}$ if

$$
\text { (i) } A A^{\oplus}=P_{A} \text { and } \quad\left(\text { ii) } \mathcal{R}\left(A^{\oplus}\right) \subseteq \mathcal{R}(A)\right. \text {, }
$$

where $P_{A}$ is the orthogonal projector onto $\mathcal{R}(A)$, and $\mathcal{R}(A)$ is the column space of $A$.

Later, the notion of the core inverse for complex matrices was extended to the ring case by Rakić, Dinčić and Djordjević [28]. The core inverse [28] of $a \in R$ is the element $x \in R$ which satisfies

$$
\text { (1) } a x a=a, \quad \text { (2) } x a x=x, \quad(3)(a x)^{*}=a x, \quad(6) x a^{2}=a \quad \text { (7) } a x^{2}=x .
$$


The element $x$ above is unique if it exists and is denoted by $a^{\boxplus}$.

Let $\delta=\{1,2,3,4,5,6,7\}$. If $x \in R$ satisfies the equations in $(i)$ for all $i \in \delta$, then $x$ is called a $\delta$-inverse of $a$. The set of all $\delta$-inverses of $a$ is denoted by $a\{\delta\}$. For example, $a\{1,2,5\}=\left\{a^{\#}\right\}$. The element $a \in R$ is regular if $a\{1\} \neq \varnothing . R^{\#}, R^{\{1,3\}}, R^{\dagger}, R^{\oplus}$ stand for the set of all group, $\{1,3\}$, Moore-Penrose, core invertible elements of $R$, respectively. Let $M_{n}(R)$ denote the ring of $n \times n$ matrices over $R$.

Given $a \in R$, the following notations will be used:

$$
a^{0}=\{x \in R: a x=0\} \text { and }{ }^{0} a=\{x \in R: x a=0\} .
$$

An element $a \in R$ is said to be EP if $a \in R^{\#} \cap R^{\dagger}$ and $a^{\#}=a^{\dagger}$. An element $a \in R$ satisfying $a^{*}=a$ is called Hermitian.

Now, we present several known characterizations for some generalized inverses, which play an important role in the sequel.

Lemma 2.1. [31, Theorem 3.1] Let $a, x \in R$. Then $a \in R^{\oplus}$ with $x=a^{\oplus}$ if and only if

$$
(a x)^{*}=a x, \quad x a^{2}=a \text { and } a x^{2}=x .
$$

Lemma 2.2. [31, Theorem 2.6] Let $a \in R$. Then $a \in R^{\oplus}$ if and only if $a \in R^{\#} \cap R^{\{1,3\}}$. In this case, $a^{\boxplus}=a^{\#} a a^{(1,3)}$, where $a^{(1,3)} \in a\{1,3\}$.

Lemma 2.3. [15, Theorem 1] Let $a \in R$. Then $a \in R^{\#}$ if and only if $a=a^{2} x=y a^{2}$ for some $x, y \in R$. In this case, $a^{\#}=y a x=y^{2} a=a x^{2}$.

Lemma 2.4. [14, p. 201] Let $a, x \in R$. Then $x$ is a $\{1,3\}$-inverse of $a$ if and only if $a=x^{*} a^{*} a$.

The following lemmas will also be useful.

Lemma 2.5. [4, Corollary 3.4] Let $a, x \in R$ with $x a=a x$ and $x a^{*}=a^{*} x$. If $a \in R^{\oplus}$, then $x a^{\oplus}=a^{\oplus} x$.

Lemma 2.6. [5, Cline's formula] Let $a, b \in R$. If $a b$ is Drazin invertible, then ba is Drazin invertible and $(b a)^{D}=b\left((a b)^{D}\right)^{2} a$.

Lemma 2.7. [28, Theorem 3.1] Let $a \in R$. Then the following are equivalent:

(i) $a$ is $E P$;

(ii) $a \in R^{\oplus}$ and $a^{\#}=a^{\oplus}$.

(iii) $a \in R^{\oplus}$ and $a a^{\oplus}=a^{\oplus} a$.

(iv) $a \in R^{\#} \cap R^{\dagger}$ and $a^{\dagger}=a^{\oplus}$

Lemma 2.8. [28, Lemma 2.5, Lemma 2.6] Let $a, b \in R$.

(i) If $a R \subseteq b R$, then ${ }^{\circ} b \subseteq{ }^{\circ} a$.

(ii) If $b$ is regular and ${ }^{\circ} b \subseteq{ }^{\circ} a$, then $a R \subseteq b R$.

(iii) If $R a \subseteq R b$, then $b^{\circ} \subseteq a^{\circ}$.

(iv) If $b$ is regular and $b^{\circ} \subseteq a^{\circ}$, then $R a \subseteq R b$. 


\section{One-sided and Two-sided Reverse Order Laws}

In this section, we will give some equivalent conditions of the one-sided $(a b)^{\oplus}=b^{\oplus} a^{\oplus}$, the two-sided reverse order laws $(a b)^{\oplus}=b^{\oplus} a^{\oplus}$ and $(b a)^{\oplus}=a^{\oplus} b^{\oplus}$ for the core inverse in rings.

First, in order to prove our main results, we give new existence criterion for the core inverse as follows:

Theorem 3.1. Let $a, x \in R$. Then the following are equivalent:

(i) $a \in R^{\oplus}$ and $x=a^{\oplus}$;

(ii) $a x a=a, x R=a R$ and $R x \subseteq R a^{*}$;

(iii) $a x a=a,{ }^{0} x={ }^{0} a$ and $\left(a^{*}\right)^{0} \subseteq x^{0}$;

(iv) $x a x=x, x R=a R$ and $R x=R a^{*}$;

(v) $x a x=x, x R=a R$ and $R a^{*} \subseteq R x$;

(vi) $x a x=x,{ }^{0} x={ }^{0} a$ and $x^{0} \subseteq\left(a^{*}\right)^{0}$;

(vii) $a \in R^{\#}$, axa $=a,(a x)^{*}=a x$, and $x R \subseteq a R$;

(viii) $a \in R^{\#}, x a x=x,(a x)^{*}=a x$, and $a R \subseteq x R$.

Proof. (i) $\Rightarrow$ (ii) and (iv) $\Rightarrow$ (v) are trivial.

(ii) $\Rightarrow$ (iii) and (v) $\Rightarrow$ (vi) follow directly from Lemma 2.8 .

(iii) $\Rightarrow$ (iv) Note that $a^{*}$ is regular. Since $\left(a^{*}\right)^{0} \subseteq x^{0}$, using Lemma 2.8, we have $R x \subseteq R a^{*}$, which implies that $x=t_{1} a^{*}$ for some $t_{1} \in R$. Thus, we get

$$
x=t_{1} a^{*}=t_{1}(a x a)^{*}=t_{1} a^{*} x^{*} a^{*}=x x^{*} a^{*}=x(a x)^{*} .
$$

Multiplying the previous equality by $a$ from the left side, we obtain $a x=a x(a x)^{*}$, which gives that $a x=(a x)^{*}$. Therefore, we get $x=x(a x)^{*}=x a x$.

From $a=a x a=(a x)^{*} a=x^{*} a^{*} a$, it follows that $a^{*}=a^{*} a x$. So, $R a^{*} \subseteq R x$. Therefore, $R a^{*}=R x$. According to the condition ${ }^{0} x={ }^{0} a$, we deduce that $x R=a R$ by Lemma 2.8.

(vi) $\Rightarrow$ (i) Clearly, we have $R a^{*} \subseteq R x$, which yields $a=x^{*} t_{2}$ for some $t_{2} \in R$. So, we have

$$
a=x^{*} t_{2}=(x a x)^{*} t_{2}=x^{*} a^{*} x^{*} t_{2}=x^{*} a^{*} a=(a x)^{*} a .
$$

Hence, we get $a x=(a x)^{*} a x$, which immediately yields $a x=(a x)^{*}$. Then, $a=(a x)^{*} a=a x a$.

On one hand, we can see that $x=x a x=x(a x)^{*}=x x^{*} a^{*}$, which implies $R x \subseteq R a^{*}$, leading to $R x=R a^{*}$, since $R a^{*} \subseteq R x$. On the other hand, it is easy to get $x R=a R$. Finally, by the definition of the core inverse, we claim that $a \in R^{\oplus}$ and $x=a^{\oplus}$.

(i) $\Rightarrow$ (vii) and (i) $\Rightarrow$ (viii) can be obtained by Lemma 2.2 and the definition of core inverse.

(vii) $\Rightarrow$ (i) Note that $x \in a\{1,3\}$ and $x=a t_{3}$ for some $t_{3} \in R$. From Lemma 2.2, it follows that $a \in R^{\oplus}$ and $a^{\boxplus}=a^{\#} a x=a^{\#} a a t_{3}=a t_{3}=x$.

(viii) $\Rightarrow$ (i) This is analogous to the proof of (ii) $\Rightarrow$ (i). Indeed, there exists $r_{4} \in R$ such that $a=x t_{4}$. Then, we have

$$
a x a=a x a a a^{\#}=a(x a x) t_{4} a^{\#}=a\left(x t_{4}\right) a^{\#}=a^{2} a^{\#}=a,
$$


which gives that $x \in a\{1,3\}$, since $(a x)^{*}=a x$. Hence, $a \in R^{\boxplus}$ with

$$
a^{\boxplus}=a^{\#} a x=a a^{\#} x=x t_{4} a^{\#} x=x a\left(x t_{4}\right) a^{\#} x=x a^{2} a^{\#} x=x a x=x .
$$

Now, we state a sufficient condition for the reverse order law $(a b)^{\oplus}=b^{\oplus} a^{\oplus}$ to hold, which extends [4, Theorem 3.5].

Theorem 3.2. Let $a, b \in R^{\oplus}, a b \in R^{\#}$. If $a b b^{\oplus}=b b^{\oplus} a$ and $b a a^{\oplus}=a a^{\oplus} b$, then $a b \in R^{\oplus}$ and $(a b)^{\oplus}=b^{\oplus} a^{\oplus}$.

Proof. According to Theorem 3.1 (i) and (ii), it suffices to prove that $a b b^{\oplus} a^{\oplus} a b=a b$, $b^{\oplus} a^{\oplus} R=a b R$, and $R b^{\oplus} a^{\oplus} \subseteq R(a b)^{*}$.

The condition $a b b^{\oplus}=b b^{\oplus a}$ implies $a^{*} b b^{\oplus}=b b^{\oplus} a^{*}$. Then, from Lemma 2.5, it follows that $a^{\boxplus} b b^{\oplus}=b b^{\oplus} a^{\boxplus}$. Similarly, we obtain $b^{\oplus a a^{\oplus}}=a a^{\oplus} b^{\oplus}$. Hence, we get $a b b^{\oplus} a^{\oplus a b}=$ $a a^{\oplus} a b b^{\oplus} b=a b$.

On one hand, we have

$$
\begin{aligned}
b^{\oplus} a^{\oplus} & =\left(b^{\oplus a} a a^{\oplus}\right) a^{\oplus}=a a^{\oplus} b^{\oplus} a^{\oplus}=a\left(a^{\oplus} b b^{\oplus}\right) b^{\oplus} a^{\oplus} \\
& =a b b^{\oplus} a^{\oplus} b^{\oplus} a^{\oplus}=a b\left(b^{\oplus} a^{\oplus}\right)^{2},
\end{aligned}
$$

which gives $b^{\oplus} a^{\oplus} R \subseteq a b R$. On the other hand, combining $b^{\oplus} a^{\oplus}=a b\left(b^{\oplus} a^{\oplus}\right)^{2}$ and $a b b^{\oplus} a^{\oplus} a b=$ $a b$, we get

$$
\begin{aligned}
a b & =(a b)^{\#} a b\left(b^{\oplus} a^{\boxplus}\right)(a b)^{2}=(a b)^{\#} a b a b\left(b^{\oplus} a^{\boxplus}\right)^{2}(a b)^{2} \\
& =\left(a b\left(b^{\oplus} a^{\boxplus}\right)^{2}\right)(a b)^{2}=b^{\oplus} a^{\oplus}(a b)^{2},
\end{aligned}
$$

which yields $a b R \subseteq b^{\oplus} a^{\oplus} R$. Thus, we have $b^{\oplus} a^{\oplus} R=a b R$.

Finally, by the following equalities

$$
\begin{aligned}
b^{\oplus} a^{\oplus} & =b^{\oplus}\left(b b^{\oplus} a^{\oplus}\right) a a^{\oplus}=b^{\oplus} a^{\oplus} b b^{\oplus a} a a^{\oplus}=b^{\oplus} a^{\oplus}\left(a a^{\oplus} b b^{\oplus}\right)^{*} \\
& =b^{\oplus} a^{\oplus}\left(a b b^{\oplus} a^{\oplus}\right)^{*}=b^{\oplus} a^{\oplus}\left(b^{\oplus} a^{\oplus}\right)^{*}(a b)^{*},
\end{aligned}
$$

we have $R b^{\oplus} a^{\oplus} \subseteq R(a b)^{*}$.

The proof is completed.

Proposition 3.3. [4, Theorem 3.5] Let $a, b \in R^{\oplus}$ with $a b=b a$ and $a b^{*}=b^{*} a$. Then $a b \in R^{\boxplus}$ and $(a b)^{\oplus}=b^{\oplus} a^{\oplus}$.

Proof. From $a b=b a$ and $a b^{*}=b^{*} a$, we have $a b^{\oplus}=b^{\oplus} a$ and $b a^{\oplus}=a^{\oplus b} b$ by Lemma 2.5, which imply $a b b^{\oplus}=b b^{\oplus} a$ and $b a a^{\oplus}=a a^{\oplus} b$. In addition, we know that $a b \in R^{\#}$, since $a, b \in R^{\#}$ with $a b=b a$. Then, $(a b)^{\oplus}=b^{\oplus} a^{\oplus}$ by Theorem 3.2.

Remark 3.4. We have seen that the condition of Proposition 3.3 can imply the condition of Theorem 3.2. But, in general, the condition of Theorem 3.2 does not imply the condition of Proposition 3.3. Indeed, take non-commutative invertible elements $a, b \in R$. Obviously, $a$ and $b$ satisfy the condition of Theorem 3.2. However, $a b \neq b a$. 
Next, we give an equivalent condition which ensures the reverse order law $(a b)^{\oplus}=b^{\oplus} a^{\oplus}$ holds.

Theorem 3.5. Let $a, b \in R^{\oplus}$. Then the following are equivalent:

(i) $a b \in R^{\oplus}$ and $(a b)^{\oplus}=b^{\oplus} a^{\oplus}$;

(ii) $a b \in R^{\#}, a b^{\oplus} R \subseteq b^{\oplus} a^{\oplus} R$, and $b^{*} a^{\#}\left(1-\left(a b b^{\oplus} a^{\oplus}\right)^{*}\right) a=0$.

Proof. (i) $\Rightarrow$ (ii) Obviously, $a b \in R^{\#}$. Note that

$$
a b^{\oplus}=a b\left(b^{\oplus}\right)^{2}=b^{\oplus} a^{\oplus}(a b)^{2}\left(b^{\oplus}\right)^{2},
$$

which implies $a b^{\oplus} R \subseteq b^{\boxplus} a^{\boxplus} R$. By Lemma 2.2, we have $a^{\boxplus} a=a^{\#} a a^{(1,3)} a=a^{\#} a$, together with $b^{*}=b^{*} b b^{\oplus}$, we obtain

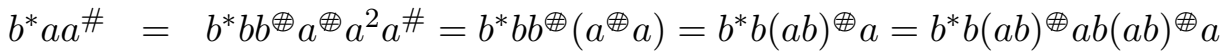

$$
\begin{aligned}
& =b^{*} b b^{\oplus} a^{\oplus} a b b^{\oplus} a^{\oplus} a=\left(b^{*} b b^{\oplus}\right)\left(a^{\oplus} a\right) b b^{\oplus} a^{\oplus} a=b^{*} a^{\#} a b b^{\oplus} a^{\oplus a} \\
& =b^{*} a^{\#}\left(a b b^{\boxplus} a^{\boxplus}\right)^{*} a \text {. }
\end{aligned}
$$

Therefore, $b^{*} a^{\#}\left(1-\left(a b b^{\oplus} a^{\boxplus}\right)^{*}\right) a=0$.

(ii) $\Rightarrow$ (i) Observe that $a^{\boxplus}=a^{\oplus} a a^{(1,3)}$ and $a^{\#} a a^{\oplus}=a^{\oplus}$ by Lemma 2.2. Then, applying the condition $b^{*} a^{\#} a=b^{*} a^{\#}\left(a b b^{\oplus} a^{\boxplus}\right)^{*} a$, we obtain

$$
\begin{aligned}
b^{*} a^{\oplus} & =\left(b^{*} a^{\#} a\right) a^{\oplus}=b^{*} a^{\#}\left(a b b^{\oplus} a^{\oplus}\right)^{*} a a^{\oplus}=b^{*} a^{\#}\left(a^{\boxplus}\right)^{*} b b^{\oplus} a^{*} a a^{\oplus} \\
& =b^{*} a^{\#}\left(a^{\oplus}\right)^{*} b b^{\oplus} a^{*}=b^{*} a^{\#}\left(a^{\oplus} a a^{(1,3)}\right)^{*} b b^{\oplus} a^{*} \\
& =b^{*} a^{\#} a a^{(1,3)}\left(a^{\oplus}\right)^{*} b b^{\oplus} a^{*} \\
& =b^{*} a^{\oplus}\left(a^{\oplus}\right)^{*} b b^{\oplus} a^{*}
\end{aligned}
$$

which yields $\left(a^{\boxplus}\right)^{*} b=a b b^{\oplus} a^{\boxplus}\left(a^{\boxplus}\right)^{*} b$. By the previous equality, we get

$$
\begin{aligned}
\left(a^{\oplus}\right)^{*}\left(b^{\oplus}\right)^{*} & =\left(a^{\oplus}\right)^{*}\left(b^{\oplus} b b^{\oplus}\right)^{*}=\left(\left(a^{\oplus}\right)^{*} b\right) b^{\oplus}\left(b^{\oplus}\right)^{*}=a b b^{\oplus} a^{\oplus}\left(a^{\oplus}\right)^{*} b b^{\oplus}\left(b^{\oplus}\right)^{*} \\
& =a b b^{\oplus} a^{\oplus}\left(a^{\oplus}\right)^{*}\left(b^{\oplus}\right)^{*}=a b b^{\oplus} a^{\oplus}\left(b^{\oplus} a^{\oplus}\right)^{*},
\end{aligned}
$$

which gives $b^{\oplus} a^{\oplus}=b^{\oplus} a^{\oplus}\left(a b b^{\oplus} a^{\oplus}\right)^{*}$. Then, $a b b^{\oplus} a^{\oplus}=a b b^{\oplus} a^{\oplus}\left(a b b^{\oplus} a^{\oplus}\right)^{*}$. So, we have $a b b^{\oplus} a^{\oplus}=\left(a b b^{\oplus} a^{\oplus}\right)^{*}$. This immediately yields $b^{\oplus} a^{\oplus}=b^{\oplus} a^{\oplus} a b b^{\oplus} a^{\oplus}$.

The condition $a b^{\oplus} R \subseteq b^{\oplus} a^{\boxplus} R$ ensures that $a b^{\oplus}=b^{\oplus} a^{\oplus} r$ for some $r \in R$. Hence, we get $a b=\left(a b^{\oplus}\right) b^{2}=b^{\oplus} a^{\oplus} r b^{2}$, which gives $a b R \subseteq b^{\oplus} a^{\oplus} R$. From Theorem 3.1, it follows that $a b \in R^{\oplus}$ and $(a b)^{\oplus}=b^{\oplus} a^{\boxplus}$.

In order to simplify the proof of the following theorems, we present a useful lemma.

Lemma 3.6. Let $a, b, a b \in R^{\oplus}$ with $(a b)^{\oplus}=b^{\oplus} a^{\oplus}$. Then

(i) $a b=b b^{\oplus} a b=b^{\oplus} b a b$;

(ii) $a b R \subseteq b a R$;

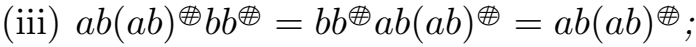

(iv) $b b^{\oplus a} a^{\oplus} \in a b b^{\oplus}\{3,6\}$. 
Proof. (i) Suppose that $(a b)^{\oplus}=b^{\oplus} a^{\oplus}$. Then, we have

$$
a b=b^{\oplus} a^{\oplus}(a b)^{2}=b b^{\oplus}\left(b^{\oplus} a^{\oplus}(a b)^{2}\right)=b b^{\oplus a} a,
$$

and

$$
a b=b^{\oplus} a^{\oplus}(a b)^{2}=b^{\oplus} b\left(b^{\oplus} a^{\oplus}(a b)^{2}\right)=b^{\oplus} b a b .
$$

(ii) From (i), it follows that

$$
a b=b b^{\oplus} a b=b b^{\oplus} a^{\oplus} a^{2} b=b(a b)^{\oplus} a^{2} b=b a b\left((a b)^{\oplus}\right)^{2} a^{2} b .
$$

Hence, $a b R \subseteq b a R$.

(iii) Clearly, we have

$$
\begin{aligned}
a b(a b)^{\oplus} b b^{\oplus} & =\left(a b(a b)^{\oplus}\right)^{*}\left(b b^{\oplus}\right)^{*}=\left(b b^{\oplus} a b(a b)^{\oplus}\right)^{*}=\left(b b^{\oplus} a b b^{\oplus} a^{\oplus}\right)^{*} \\
& =\left(a b b^{\oplus} a^{\oplus}\right)^{*}=a b(a b)^{\oplus},
\end{aligned}
$$

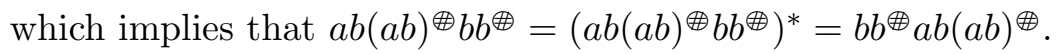

(iv) Since $(a b)^{\oplus}=b^{\oplus} a^{\oplus}$, we have $a b b^{\oplus} b b^{\oplus} a^{\oplus}=a b b^{\oplus} a^{\oplus}=a b(a b)^{\oplus}$, which gives $b b^{\oplus} a^{\oplus} \in$ $a b b^{\oplus}\{3\}$. Also, we have

$$
\begin{aligned}
b b^{\oplus} a^{\oplus}\left(a b b^{\oplus}\right)^{2} & =b\left(b^{\oplus} a^{\oplus} a b b^{\oplus} a^{\oplus}\right) a^{2} b b^{\oplus}=b b^{\oplus}\left(a^{\oplus} a^{2}\right) b b^{\oplus}=\left(b b^{\oplus a} a b b^{\oplus}\right. \\
& =a b b^{\oplus} .
\end{aligned}
$$

Thus, $b b^{\oplus} a^{\oplus} \in a b b^{\oplus}\{3,6\}$.

Deng [10] and Mosić [23] studied the reverse order law $(a b)^{\#}=b^{\#} a^{\#}$ for the group inverse under the condition $b a=a^{2}$. Motivated by this, we will consider the one-sided reverse law for the core inverse under the same condition, using Theorem 3.5.

Theorem 3.7. Let $a, b \in R^{\oplus}$ with $b a=a^{2}$. Then,

(i) $a b \in R^{\oplus}$ and $(a b)^{\oplus}=b^{\oplus} a^{\oplus}$.

(ii) $a b b^{\oplus} \in R^{\oplus}$ and $\left(a b b^{\oplus}\right)^{\oplus}=b b^{\oplus} a^{\boxplus}$.

Proof. (i) First, we prove $a b \in R^{\#}$. Note that $a \in R^{\#}$ and $b a=a^{2}$. Then, we have

$$
a b=a a^{2} a^{2}\left(a^{\#}\right)^{4} b=a b a b a\left(a^{\#}\right)^{4} b=(a b)^{2}\left(a^{\#}\right)^{3} b
$$

and

$$
a b=a^{2} a^{\#} b=b a a^{\#} b=b\left(a^{\#}\right)^{3} a a^{2} b=b\left(a^{\#}\right)^{3} a b a b=b\left(a^{\#}\right)^{3}(a b)^{2},
$$

which imply $a b \in R^{\#}$ by Lemma 2.3 .

Next, we see that

$$
a=a^{2} a^{\#}=b a a^{\#}=b^{\oplus} b^{2} a a^{\#}=b^{\oplus} b(b a) a^{\#}=b^{\oplus} b a^{2} a^{\#}=b^{\oplus} b a=b^{\oplus} a^{2} .
$$


Hence, $a b^{\oplus}=b^{\oplus} a^{2} b^{\oplus}=b^{\oplus} a^{\oplus} a^{3} b^{\oplus}$, which yields $a b^{\oplus} R \subseteq b^{\oplus} a^{\oplus} R$. Note that $a=b^{\oplus} b a$, then we deduce that $a^{\oplus}=a\left(a^{\oplus}\right)^{2}=b^{\oplus} b a\left(a^{\oplus}\right)^{2}=b^{\oplus b a^{\oplus}}$. In addition, the assumption $b a=a^{2}$ ensures that $a a^{\oplus}=a^{2}\left(a^{\boxplus}\right)^{2}=b a\left(a^{\boxplus}\right)^{2}=b a^{\oplus}$. Therefore,

$$
a b b^{\oplus} a^{\oplus}=a b b^{\oplus} b^{\oplus} b a^{\boxplus}=a b^{\oplus} b a^{\boxplus}=a\left(b^{\oplus} a\right) a^{\boxplus}=a\left(b^{\oplus} a^{2}\right) a^{\#} a^{\boxplus}=a a a^{\#} a^{\boxplus}=a a^{\oplus},
$$

which implies $\left(a b b^{\oplus} a^{\boxplus}\right)^{*}=a b b^{\oplus} a^{\boxplus}$. So, we conclude that

$$
b^{*} a^{\#}\left(1-\left(a b b^{\oplus} a^{\boxplus}\right)^{*}\right) a=b^{*} a^{\#} a-b^{*} a^{\#}\left(a b b^{\oplus} a^{\boxplus}\right) a=b^{*} a^{\#} a-b^{*} a^{\#} a a^{\boxplus} a=0 .
$$

By Theorem 3.5, we claim that $a b \in R^{\oplus}$ and $(a b)^{\oplus}=b^{\oplus} a^{\oplus}$.

(ii) By (i), Lemma 3.6(iv), and Lemma 2.1, we only need to prove $b b^{\oplus} a^{\oplus} \in a b b^{\oplus}\{7\}$.

In the proof of (i), we obtain $a^{\boxplus}=b^{\oplus} b a^{\oplus}$ and $b a^{\oplus}=a a^{\oplus}$ which imply that $b b^{\oplus} a^{\oplus}=$ $b b^{\oplus} b^{\oplus}\left(b a^{\oplus}\right)=b^{\oplus a a^{\oplus}}$. Hence,

$$
b b^{\oplus} a^{\oplus}\left(a b b^{\oplus}\right)^{2}=b^{\oplus}\left(a a^{\oplus} a\right)\left(b b^{\oplus} a b\right) b^{\oplus}=b^{\oplus} a a b b^{\oplus}=\left(b^{\oplus b a b}\right) b^{\oplus}=a b b^{\oplus} .
$$

Using Lemma 3.6 and Theorem 3.1, we deduce the following result.

Theorem 3.8. Let $a, b, a b \in R^{\boxplus}$. Then the following are equivalent:

(i) $(a b)^{\oplus}=b^{\oplus} a^{\oplus}$;

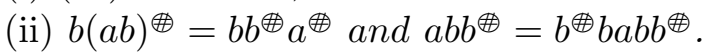

Proof. (i) $\Rightarrow$ (ii) can be obtained by Lemma 3.6(i).

(ii) $\Rightarrow$ (i) Since $b(a b)^{\oplus}=b b^{\oplus} a^{\oplus}$, we have

$$
a\left(b b^{\oplus} a^{\boxplus}\right) a b=a b(a b)^{\oplus} a b=a b \quad \text { and } a b b^{\oplus} a^{\oplus}=a b(a b)^{\oplus},
$$

which implies $b^{\oplus} a^{\oplus} \in a b\{1,3\}$. In addition, observe that

$$
\begin{aligned}
& b^{\oplus} a^{\oplus}=b^{\oplus}\left(b b^{\oplus} a^{\oplus}\right)=b^{\oplus b} b(a b)^{\oplus}=b^{\oplus} b a b\left((a b)^{\oplus}\right)^{2} \\
& =\left(b^{\oplus} b a b b^{\oplus}\right) b\left((a b)^{\oplus}\right)^{2}=a b b^{\oplus} b\left((a b)^{\oplus}\right)^{2},
\end{aligned}
$$

which yields $b^{\oplus} a^{\oplus} R \subseteq a b R$. Hence, by Theorem 3.1 (i) and (vii), we obtain $(a b)^{\oplus}=b^{\oplus} a^{\oplus}$.

In the following theorem, we investigate the one-sided reverse order law for the core inverse under the condition $R b^{*} a \subseteq R a b^{*}$.

Theorem 3.9. Let $a, b \in R^{\oplus}$ with $R b^{*} a \subseteq R a b^{*}$. Then the following are equivalent:

(i) $a b \in R^{\oplus}$ and $(a b)^{\oplus}=b^{\oplus} a^{\oplus}$;

(ii) $a b \in R^{\#}, a b R \subseteq b a R, b^{\oplus} a R \subseteq a b R$, and $a a^{\oplus} b b^{\oplus}=b b^{\oplus a} a a^{\oplus}$;

(iii) $a b, a b b^{\oplus} \in R^{\oplus}$ with $\left(a b b^{\oplus}\right)^{\oplus}=b b^{\oplus} a^{\boxplus}$ and $(a b)^{\oplus}=b^{\oplus}\left(a b b^{\oplus}\right)^{\oplus}$. 
Proof. (i) $\Rightarrow$ (ii) Obviously, $a b \in R^{\#}$. From $(a b)^{\oplus}=b^{\oplus} a^{\oplus}$ and Lemma 3.6, it follows that $a b R \subseteq b a R$. The equality $b^{\oplus} a=b^{\oplus} a^{\oplus} a^{2}=a b\left((a b)^{\oplus}\right)^{2} a^{2}$ implies $b^{\oplus} a R \subseteq a b R$.

Next, our aim is to prove $a a^{\oplus} b b^{\oplus}=b b^{\oplus} a a^{\oplus}$. The assumption $R b^{*} a \subseteq R a b^{*}$ guarantees $b^{*} a=w_{1} a b^{*}$ for some $w_{1} \in R$. Then, we have

$$
b^{*} a=w_{1} a\left(b b^{\oplus} b\right)^{*}=w_{1} a b^{*} b b^{\oplus}=b^{*} a b b^{\oplus} .
$$

Note that $a b=b b^{\oplus} a b$ by Lemma 3.6(i), we obtain

$$
\begin{aligned}
a b b^{\oplus} a^{\oplus} & =b b^{\oplus} a b b^{\oplus} a^{\oplus}=\left(b b^{\oplus}\right)^{*} a b b^{\oplus} a^{\oplus}=\left(b^{\oplus}\right)^{*}\left(b^{*} a b b^{\oplus}\right) a^{\oplus} \\
& =\left(b^{\oplus}\right)^{*} b^{*} a a^{\oplus}=b b^{\oplus} a a^{\oplus} .
\end{aligned}
$$

Therefore, the following equalities hold:

$$
a b b^{\oplus} a^{\boxplus}=\left(a b b^{\oplus a} a^{\boxplus}\right)^{*}=\left(b b^{\oplus} a a^{\oplus}\right)^{*}=a a^{\boxplus} b b^{\oplus} .
$$

So, we have $a a^{\oplus b b^{\oplus}}=b b^{\oplus a a^{\boxplus}}$.

(ii) $\Rightarrow$ (i) Since $a b R \subseteq b a R$, there exists $w_{2} \in R$ such that $a b=b a w_{2}$. Therefore, we obtain $a b=b b^{\oplus}\left(b a w_{2}\right)=b b^{\oplus} a b$. Then, the equality $a b b^{\oplus} a^{\oplus}=b b^{\oplus} a a^{\oplus}$ can be obtained in a similar way as in the proof of (i) $\Rightarrow$ (ii). Hence, we deduce that

$$
\begin{aligned}
& \left(b^{\boxplus} a^{\boxplus}\right)^{*}(a b)^{*} a b=\left(a b b^{\oplus} a^{\boxplus}\right)^{*} a b=\left(b b^{\oplus} a a^{\boxplus}\right)^{*} a b=a a^{\oplus} b b^{\oplus} a b \\
& =b b^{\oplus a a^{\oplus}} a b=b b^{\oplus} a b=a b,
\end{aligned}
$$

which implies $b^{\oplus} a^{\oplus} \in a b\{1,3\}$ by Lemma 2.4 .

From $b^{\oplus} a R \subseteq a b R$, we know that $b^{\oplus} a=a b w_{3}$ for some $w_{3} \in R$. Then, we obtain $b^{\oplus} a^{\oplus}=b^{\oplus a} a\left(a^{\boxplus}\right)^{2}=a b w_{3}\left(a^{\oplus}\right)^{2}$, which gives that $b^{\oplus} a^{\oplus} R \subseteq a b R$. Hence, $a b \in R^{\oplus}$ with $(a b)^{\oplus}=b^{\oplus} a^{\oplus}$ by Theorem 3.1.

(i) $\Rightarrow$ (iii) According to the condition (i) and Lemma 3.6 (iv), we get $b b^{\oplus} a^{\oplus} \in a b b^{\oplus}\{3,6\}$. In addition, from the proof of (i) $\Rightarrow$ (ii), we see that $a b b^{\oplus} a^{\oplus}=b b^{\oplus a a^{\oplus}}=a a^{\oplus} b b^{\oplus}$. Then, we obtain

$$
\begin{aligned}
& a b b^{\oplus}\left(b b^{\oplus} a^{\boxplus}\right)^{2}=\left(a b b^{\oplus} a^{\boxplus}\right) b b^{\oplus} a^{\oplus}=a a^{\oplus} b b^{\oplus} b b^{\oplus} a^{\oplus}=a a^{\oplus b} b b^{\oplus} a^{\oplus} \\
& =b b^{\oplus} a a^{\oplus} a^{\oplus}=b b^{\oplus a} a^{\oplus} .
\end{aligned}
$$

By Lemma 2.1, it follows that $a b b^{\oplus} \in R^{\oplus}$ and $\left(a b b^{\oplus}\right)^{\oplus}=b b^{\oplus} a^{\oplus}$. Then, it is easy to see that $(a b)^{\oplus}=b^{\oplus}\left(b b^{\oplus} a^{\oplus}\right)=b^{\oplus}\left(a b b^{\oplus}\right)^{\oplus}$.

(iii) $\Rightarrow$ (i) Suppose that $\left(a b b^{\oplus}\right)^{\oplus}=b b^{\oplus} a^{\oplus}$ and $(a b)^{\oplus}=b^{\oplus}\left(a b b^{\oplus}\right)^{\oplus}$. Then, we deduce

$$
(a b)^{\oplus}=b^{\oplus}\left(a b b^{\oplus}\right)^{\oplus}=b^{\oplus} b b^{\oplus a^{\oplus}}=b^{\oplus} a^{\oplus} .
$$

Remark 3.10. (1) Note that Corollary 3.3 can also be obtained by Theorem 3.9. Indeed, we have $a b^{\oplus}=b^{\oplus} a, b a^{\oplus}=a^{\oplus} b$, and $a^{\oplus} b^{\oplus}=b^{\oplus} a^{\oplus}$ by Lemma 2.5. Then, $a a^{\oplus b b^{\oplus}}=b b^{\oplus} a a^{\oplus}$ and $b^{\oplus a}=a b^{\oplus}=a b\left(b^{\oplus}\right)^{2}$, which gives $b^{\oplus a} R \subseteq a b R$. Hence, we have $a b \in R^{\oplus}$ and $(a b)^{\boxplus}=b^{\oplus} a^{\oplus}$ by Theorem 3.9. 
(2) In general, any item of (i)-(iii) in Theorem 3.9 does not imply $R b^{*} a \subseteq R a b^{*}$. For example, let $\mathbb{Z}_{2}$ be the ring of integers modulo 2 , take $R=M_{2}\left(\mathbb{Z}_{2}\right)$ with the transpose of matrices as involution. Setting $a=b=\left[\begin{array}{ll}1 & 1 \\ 0 & 0\end{array}\right] \in R$. Then $a^{2}=a$, which implies that $a \in R^{\#}$ with $a^{\#}=a$. Note that $a=\left[\begin{array}{ll}1 & 0 \\ 0 & 0\end{array}\right] a^{*} a \in R a^{*} a$, then $a \in R^{\{1,3\}}$. Hence, $a \in R^{\boxplus}$ with $a^{\boxplus}=\left[\begin{array}{ll}1 & 0 \\ 0 & 0\end{array}\right]$. Then, it is easy to check that such $a$ and $b$ satisfy any item of (i) - (iii) in Theorem 3.9. However, $a b^{*}=0$ and $b^{*} a \neq 0$, so $R b^{*} a \nsupseteq R a b^{*}$.

(3) If we replace $R b^{*} a \subseteq R a b^{*}$ with $b a=a^{2}$ in Theorem 3.9 then the items (i),(ii), and (iii) hold. Indeed, by Theorem 3.7 and Lemma 3.6, we only need to show that $b^{\oplus} a R \subseteq a b R$ and $a a^{\oplus b} b b^{\oplus}=b b^{\oplus a a^{\oplus}}$. Since $(a b)^{\oplus}=b^{\oplus} a^{\oplus}$ by Theorem 3.7, then $b^{\oplus a} R=\left(b^{\oplus} a^{\oplus}\right) a^{2} R=$ $(a b)^{\oplus} a^{2} R=a b\left((a b)^{\oplus}\right)^{2} a^{2} R \subseteq a b R$. In addition, from the proof of Theorem 3.7, we see that $a^{\oplus}=b^{\oplus} b a^{\boxplus}$ and $a a^{\oplus}=b a^{\boxplus}$, which gives $b\left(b^{\oplus a a^{\oplus}}\right)=b a^{\boxplus}=a a^{\boxplus}$ is Hermitian. Thus, we get $b b^{\oplus a} a a^{\oplus}=\left(b b^{\oplus a} a a^{\oplus}\right)^{*}=\left(a a^{\oplus}\right)^{*}\left(b b^{\oplus}\right)^{*}=a a^{\oplus} b b^{\oplus}$.

Next, we continue to consider the one-sided reverse law for the core inverse under certain conditions.

Theorem 3.11. Let $a, b \in R^{\oplus}$ with $R b \subseteq R a b$ (or $a R \subseteq a b R$ ). If $a$ is $E P$, then the following are equivalent:

(i) $a b \in R^{\oplus}$ and $(a b)^{\oplus}=b^{\oplus} a^{\oplus}$;

(ii) $\left(a^{\oplus}\right)^{*} b \in R^{\oplus}$ and $\left(\left(a^{\oplus}\right)^{*} b\right)^{\oplus}=b^{\oplus} a^{*}$.

Proof. (i) $\Rightarrow$ (ii) Since $(a b)^{\oplus}=b^{\oplus} a^{\oplus}$, the we have that

$$
\left(a^{\boxplus}\right)^{*} b b^{\boxplus} a^{*}=\left(a^{\boxplus}\right)^{*}\left(b b^{\oplus}\right)^{*} a^{*}=\left(a b b^{\oplus} a^{\boxplus}\right)^{*}=a b b^{\oplus} a^{\boxplus}
$$

is Hermitian, i.e., $b^{\oplus} a^{*} \in\left(a^{\oplus}\right)^{*} b\{3\}$.

Note that $a$ is EP, then $a^{\oplus} a=a a^{\oplus}$ is Hermitian by Lemma 2.7. So, $a^{*}=\left(a\left(a^{\oplus} a\right)\right)^{*}=$ $a^{\oplus} a a^{*}$. Thus, we have

$$
\begin{aligned}
\left(a^{\oplus}\right)^{*} b\left(b^{\oplus} a^{*}\right)^{2} & =\left(\left(a^{\oplus}\right)^{*}\left(b b^{\oplus}\right)^{*} a^{*}\right) b^{\oplus} a^{*}=\left(a b b^{\oplus} a^{\oplus}\right) b^{\oplus} a^{*} \\
& =\left(a b b^{\oplus} a^{\oplus} b^{\oplus} a^{\oplus}\right) a a^{*}=b^{\oplus} a^{\oplus a} a a^{*}=b^{\oplus} a^{*} .
\end{aligned}
$$

If $R b \subseteq R a b$, then $b=x a b$ for some $x \in R$. Therefore, we can deduce that

$$
\begin{aligned}
& b^{\oplus} a^{*}\left(\left(a^{\oplus}\right)^{*} b\right)^{2}=b^{\oplus} a^{*}\left(a^{\oplus}\right)^{*} b\left(a^{\boxplus}\right)^{*} b=b^{\oplus}\left(a^{\oplus a} a\right)^{*} b\left(a^{\oplus}\right)^{*} b \\
& =b^{\oplus} a^{\oplus a} a b\left(a^{\oplus}\right)^{*}\left(b b^{\oplus}\right)^{*} b=b^{\oplus} a^{\oplus} a b\left(b b^{\oplus} a^{\boxplus}\right)^{*} b \\
& =b^{\oplus} a^{\oplus} a b\left(x a b b^{\oplus} a^{\oplus}\right)^{*} b=\left(b^{\oplus} a^{\boxplus} a b a b\right) b^{\boxplus} a^{\oplus} x^{*} b \\
& =a b b^{\oplus} a^{\oplus} x^{*} b=\left(a b b^{\oplus} a^{\oplus}\right)^{*} x^{*} b \\
& =\left(x a b b^{\oplus} a^{\oplus}\right)^{*} b=\left(b b^{\oplus} a^{\oplus}\right)^{*} b \\
& =\left(a^{\boxplus}\right)^{*}\left(b b^{\oplus}\right)^{*} b=\left(a^{\boxplus}\right)^{*} b .
\end{aligned}
$$

If $a R \subseteq a b R$, then $a=a b y$ for some $y \in R$. Thus, we get

$$
\begin{aligned}
& b^{\boxplus} a^{*}\left(\left(a^{\boxplus}\right)^{*} b\right)^{2}=b^{\boxplus} a^{\oplus} a b\left(a^{\boxplus}\right)^{*} b=b^{\oplus} a^{\boxplus} a b\left(a^{\boxplus} a a^{\boxplus}\right)^{*} b \\
& =b^{\oplus} a^{\boxplus} a b a a^{\oplus}\left(a^{\boxplus}\right)^{*} b=\left(b^{\oplus} a^{\oplus a b a b}\right) y a^{\boxplus}\left(a^{\boxplus}\right)^{*} b \\
& =a b y a^{\boxplus}\left(a^{\boxplus}\right)^{*} b=a a^{\boxplus}\left(a^{\boxplus}\right)^{*} b=\left(a^{\boxplus}\right)^{*} b .
\end{aligned}
$$


Finally, from Lemma 2.1, it follows that $\left(a^{\oplus}\right)^{*} b \in R^{\oplus}$ and $\left(\left(a^{\oplus}\right)^{*} b\right)^{\oplus}=b^{\oplus} a^{*}$.

(ii) $\Rightarrow$ (i) By Lemma 2.7, we have $a^{\#}=a^{\oplus}$. Let $c=\left(a^{\oplus}\right)^{*}$, then $c=\left(a^{\#}\right)^{*}=\left(a^{*}\right)^{\#}$, which implies that $a^{*}=c^{\#}$. Note that $c$ is EP. Thus, $a^{*}=c^{\boxplus}$. According to the condition (ii), we obtain that $c b \in R^{\oplus}$ and $(c b)^{\oplus}=b^{\oplus} c^{\oplus}$.

If $R b \subseteq R a b$, then $R b \subseteq R a b=R a a^{\oplus} a b=R a\left(a^{\oplus a} a\right)^{*} b=R a a^{*}\left(a^{\oplus}\right)^{*} b \subseteq R\left(a^{\oplus}\right)^{*} b=R c b$.

If $a R \subseteq a b R$, then $a=a b y$ for some $y \in R$. Since $\left(\left(a^{\oplus}\right)^{*} b\right)^{\oplus}=b^{\oplus} a^{*}$, then $b^{\oplus} a^{*} R=$ $\left(a^{\oplus}\right)^{*} b R$, which yields $\left(a^{\boxplus}\right)^{*} b=b^{\oplus} a^{*} z$ for some $z \in R$. Note that $a^{*}=a^{\boxplus} a a^{*}$. Then, we obtain

$$
\begin{aligned}
& \left(a^{\boxplus}\right)^{*}=\left(a a^{\oplus} a^{\boxplus}\right)^{*}=\left(a^{\oplus}\right)^{*}\left(a^{\boxplus}\right)^{*} a^{*}=\left(a^{\oplus}\right)^{*}\left(a^{\oplus}\right)^{*} a^{\boxplus} a a^{*} \\
& =\left(a^{\boxplus}\right)^{*}\left(a^{\boxplus}\right)^{*} a^{\boxplus} a b y a^{*}=\left(a^{\boxplus}\right)^{*}\left(\left(a^{\boxplus}\right)^{*}\left(a^{\boxplus} a\right)^{*}\right) b y a^{*} \\
& =\left(a^{\oplus}\right)^{*}\left(\left(a^{\boxplus}\right)^{*} b\right) y a^{*}=\left(a^{\boxplus}\right)^{*} b^{\oplus} a^{*} z y a^{*} \\
& =\left(a^{\oplus}\right)^{*} b\left(b^{\oplus}\right)^{2} a^{*} z y a^{*},
\end{aligned}
$$

which gives $\left(a^{\oplus}\right)^{*} R \subseteq\left(a^{\oplus}\right)^{*} b R$, i.e., $c R \subseteq c b R$.

Now, replacing $a$ with $c$ in the proof of (i) $\Rightarrow$ (ii), we can obtain that (i) holds.

If we replace the assumption $R b \subseteq R a b$ (or $a R \subseteq a b R$ ) of Theorem 3.11 by $R b \subseteq R a b$ and $a R \subseteq a b R$, then we obtain the following result.

Theorem 3.12. Let $a, b \in R^{\oplus}$ with $R b \subseteq R a b$ and $a R \subseteq a b R$. If $a$ is $E P$, then the following are equivalent:

(i) $a b \in R^{\oplus}$ and $(a b)^{\oplus}=b^{\oplus} a^{\oplus}$;

(ii) $a b, a b b^{\oplus} \in R^{\oplus}$ with $(a b)^{\oplus}=b^{\oplus}\left(a b b^{\oplus}\right)^{\oplus}$ and $\left(a b b^{\oplus}\right)^{\oplus}=b b^{\oplus} a^{\oplus}$.

Proof. (i) $\Rightarrow$ (ii) In order to prove $\left(a b b^{\oplus}\right)^{\boxplus}=b b^{\oplus} a^{\oplus}$, it suffices to prove that $a b b^{\oplus}\left(b b^{\oplus} a^{\boxplus}\right)^{2}=$ $b b^{\oplus} a^{\oplus}$ by Lemma 2.6 (iv).

Since $R b \subseteq R a b$ and $a R \subseteq a b R$, there exist $t_{1}, t_{2}$ such that $b=t_{1} a b$ and $a=a b t_{2}$. Note that $a b=b b^{\oplus} a b$ and $a a^{\oplus}=a^{\oplus} a$. Thus, we get

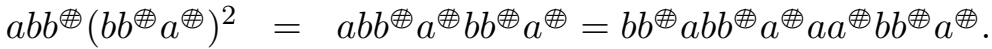

$$
\begin{aligned}
& =b b^{\oplus}\left(a b b^{\oplus} a^{\oplus a} a\right) t_{2} a^{\oplus} b b^{\oplus} a^{\oplus}=t_{1} a b b^{\oplus}\left(a b t_{2}\right) a^{\oplus b} b b^{\oplus} a^{\boxplus} \\
& =t_{1} a b b^{\oplus a} a a^{\oplus} b b^{\oplus} a^{\oplus}=t_{1}\left(a b b^{\oplus} a^{\oplus a} a b\right) b^{\oplus} a^{\oplus} \\
& =\left(t_{1} a b\right) b^{\oplus} a^{\oplus}=b b^{\oplus} a^{\oplus} \text {. }
\end{aligned}
$$

(ii) $\Rightarrow$ (i) It is obvious.

Now, necessary and sufficient conditions of the two-sided reverse laws for the core inverse are stated as follows.

Theorem 3.13. Let $a, b \in R^{\oplus}$. Then the following are equivalent:

(i) $a b, b a \in R^{\oplus}$ with $(a b)^{\oplus}=b^{\oplus} a^{\oplus},(b a)^{\oplus}=a^{\oplus} b^{\oplus}$;

(ii) $a b b^{\oplus}, b a a^{\oplus} \in R^{\oplus}$ with $\left(a b b^{\oplus}\right)^{\oplus}=b b^{\oplus} a^{\oplus},\left(b a a^{\oplus}\right)^{\oplus}=a a^{\oplus} b^{\oplus}, a b R=(a b)^{2} R$, and $b a R=(b a)^{2} R$. 
Proof. (i) $\Rightarrow$ (ii) Obviously, $a b R=(a b)^{2} R$ and $b a R=(b a)^{2} R$. By symmetry, we only need

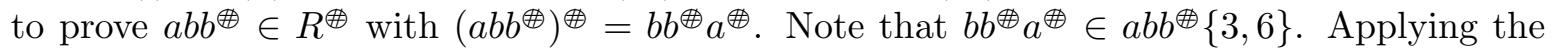
hypothesis $(b a)^{\oplus}=a^{\oplus} b^{\oplus}$ and Lemma 3.6(ii), we have $b a R=a b R$, i.e., $b a=a b u$ for some $u \in R$. Then,

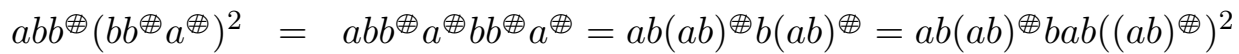

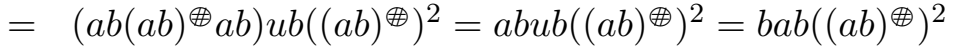

$$
\begin{aligned}
& =b(a b)^{\oplus}=b b^{\oplus} a^{\oplus} .
\end{aligned}
$$

Using Lemma 2.1, we get $a b b^{\oplus} \in R^{\oplus}$ with $\left(a b b^{\oplus)}=b b^{\oplus} a^{\oplus}\right.$.

(ii) $\Rightarrow$ (i) Since $\left(a b b^{\oplus}\right)^{\oplus}=b b^{\oplus} a^{\oplus}$, we claim that

$$
b^{\oplus} a^{\oplus} a b b^{\oplus} a^{\oplus}=b^{\oplus}\left(b b^{\oplus} a^{\oplus} a b b^{\oplus} b b^{\oplus} a^{\oplus}\right)=b^{\oplus} b b^{\oplus} a^{\oplus}=b^{\oplus} a^{\oplus}
$$

and

$$
a b b^{\oplus} a^{\oplus}=a b b^{\oplus} b b^{\oplus} a^{\boxplus}=a b b^{\oplus}\left(a b b^{\oplus}\right)^{\oplus},
$$

which imply $b^{\oplus} a^{\oplus} \in a b\{2,3\}$. In addition, observe that

$$
a b b^{\boxplus}=b b^{\oplus a} a^{\oplus}\left(a b b^{\oplus}\right)^{2}=b^{\oplus b} b\left(b b^{\oplus} a^{\oplus}\left(a b b^{\oplus}\right)^{2}\right)=b^{\oplus} b a b b^{\oplus} .
$$

Multiplying the previous equality by $b$ from the right side, we get $a b=b^{\oplus} b a b$. Symmetrically, $b a=a^{\boxplus} a b a$. Hence, we have $a b=b^{\oplus}(b a) b=b^{\oplus} a^{\boxplus}(a b)^{2}$, which gives $a b R \subseteq b^{\oplus} a^{\boxplus} R$ and $R a b=R(a b)^{2}$. Note that $a b R=(a b)^{2} R$, then $a b \in R^{\#}$ by Lemma 2.3. Finally, from Theorem 3.1, it follows that $a b \in R^{\oplus}$ with $(a b)^{\oplus}=b^{\oplus} a^{\oplus}$. The statement for $b a$ can be obtained by symmetry.

Recall that a ring $R$ (with unit 1 ) is a Dedekind-finite ring if $a b=1$ is sufficient for $b a=1$. Let $p=q=1 \in R$ in [27, Theorem 1]. Then we can see the fact: If $a \in R$ is regular, $a^{-} \in a\{1\}$, then $u=a^{2} a^{-}+1-a a^{-}$is right (resp. left) invertible if and only if $a R=a^{2} R$ (resp. $R a=R a^{2}$ ). Form the previous fact, it immediately yields that $a R=a^{2} R$ if and only if $R a=R a^{2}$, where $R$ is a Dedekind-finite ring, and $a$ is regular in $R$. Thus, by Theorem 3.13, we get

Corollary 3.14. Let $R$ be a Dedekind-finite ring and $a, b \in R^{\boxplus}$. Then the following are equivalent:

(i) $a b, b a \in R^{\oplus}$ with $(a b)^{\oplus}=b^{\oplus a^{\oplus}},(b a)^{\oplus}=a^{\oplus b^{\oplus}}$;

(ii) $a b b^{\oplus}, b a a^{\oplus} \in R^{\oplus}$ with $\left(a b b^{\oplus}\right)^{\oplus}=b b^{\oplus} a^{\oplus},\left(b a a^{\oplus}\right)^{\oplus}=a a^{\oplus} b^{\oplus}$.

Proof. According to the proof of Theorem 3.13, we only need to prove that $a b$ and $b a$ are regular. Suppose that $\left(a b b^{\oplus)}=b b^{\oplus} a^{\oplus}\right.$, then we have

$$
a b b^{\oplus}=b b^{\oplus} a^{\oplus}\left(a b b^{\oplus}\right)^{2}=b b^{\oplus}\left(b b^{\oplus a} a^{\oplus}\left(a b b^{\oplus}\right)^{2}\right)=b b^{\oplus} a b b^{\oplus},
$$

which yields

$$
a b=\left(a b b^{\oplus}\right) b=b b^{\oplus} a b b^{\oplus} b=b b^{\oplus} a b=\left(b b^{\oplus} a^{\oplus}\right) a^{2} b=a b b^{\oplus}\left(b b^{\oplus} a^{\oplus}\right)^{2} a a b .
$$

Therefore, $a b$ is regular. Similarly, by the condition $\left(b a a^{\oplus}\right)^{\oplus}=a a^{\oplus} b^{\oplus}$, we can obtain that $b a$ is regular. 


\section{Mixed-type Reverse Order Laws}

In this section, we will consider necessary and sufficient conditions for the mixed-type reverse laws: $(a b)^{\#}=b^{\oplus}\left(a b b^{\oplus}\right)^{\oplus}, a^{\boxplus}=b(a b)^{\#}$ and $(a b)^{\#}=b^{\oplus} a^{\oplus}$ to hold in rings.

Theorem 4.1. Let $b \in R^{\oplus}, a b b^{\oplus} \in R^{\oplus}$. Then the following are equivalent:

(i) $a b \in R^{\oplus}$ and $(a b)^{\oplus}=(a b)^{\#}=b^{\oplus}\left(a b b^{\oplus}\right)^{\oplus}$;

(ii) $b^{\oplus}\left(a b b^{\oplus}\right) \oplus \in a b\{5\}$;

(iii) $b^{\oplus} b a b=a b=b b^{\oplus} a b$ and $b a\left(a b b^{\oplus}\right)=\left(a b b^{\oplus}\right)^{\oplus} a b$;

(iv) $a b \in R^{\#}$ and $\left(a b b^{\oplus)}=b(a b)^{\#}\right.$.

Proof. (i) $\Rightarrow$ (ii) It is obvious.

(ii) $\Rightarrow$ (iii) Note that $a b b^{\oplus}\left(a b b^{\oplus}\right)^{\oplus a} a b=\left(a b b^{\oplus}\left(a b b^{\oplus}\right)^{\oplus a b b^{\oplus}}\right) b=a b b^{\oplus} b=a b$. Also, since $b^{\oplus}\left(a b b^{\oplus}\right)^{\oplus} \in a b\{5\}$, we have $a b=b^{\oplus}\left(a b b^{\oplus}\right)^{\oplus}(a b)^{2}$. From the previous equality, we get

$$
a b=b b^{\oplus}\left(b^{\oplus}\left(a b b^{\oplus}\right)^{\oplus}(a b)^{2}\right)=b b^{\oplus a b}
$$

and

$$
a b=b^{\oplus} b\left(b^{\oplus}\left(a b b^{\oplus}\right) \oplus(a b)^{2}\right)=b^{\oplus} b a b .
$$

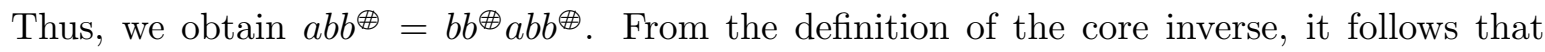
$\left(a b b^{\oplus}\right)^{\oplus} R=a b b^{\oplus} R$, i.e., $\left(a b b^{\boxplus}\right)^{\oplus}=a b b^{\oplus} u$ for some $u \in R$. Hence, we deduce

$$
\left(a b b^{\oplus}\right)^{\oplus}=b b^{\oplus} a b b^{\oplus} u=b b^{\oplus}\left(a b b^{\oplus}\right)^{\oplus} .
$$

Thus, the following equations hold:

$$
b a\left(a b b^{\oplus}\right)^{\oplus}=b a b b^{\oplus}\left(a b b^{\oplus}\right)^{\oplus}=b b^{\oplus}\left(a b b^{\oplus}\right)^{\oplus} a b=\left(a b b^{\oplus}\right)^{\oplus a} a b .
$$

(iii) $\Rightarrow$ (iv) Clearly, $b^{\oplus}\left(a b b^{\oplus}\right) \oplus \in a b\{1,2\}$.

Now, we prove $b^{\oplus}\left(a b b^{\oplus}\right)^{\oplus} \in a b\{5\}$. Since $a b=b b^{\oplus} a b$, from the proof of (ii) $\Rightarrow$ (iii), we can get $\left(a b b^{\oplus)}=b b^{\oplus}\left(a b b^{\oplus}\right)^{\oplus}\right.$. Then,

$$
(a b) b^{\oplus}\left(a b b^{\oplus}\right)^{\oplus}=b^{\oplus} b a b b^{\oplus}\left(a b b^{\oplus}\right)^{\oplus}=b^{\oplus b a}\left(a b b^{\oplus}\right)^{\oplus}=b^{\oplus}\left(a b b^{\oplus}\right)^{\oplus}(a b),
$$

which implies that $(a b)^{\#}=b^{\oplus}\left(a b b^{\boxplus}\right)^{\oplus}$. Thus, we get

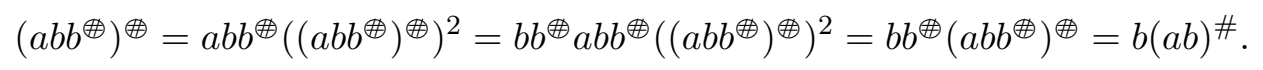

(iv) $\Rightarrow$ (i) Since $\left(a b b^{\oplus)}=b(a b)^{\#}\right.$, we have

$$
\begin{aligned}
a b & =a b b^{\oplus} b=\left(a b b^{\oplus}\right)^{\oplus}\left(a b b^{\oplus}\right)^{2} b=b(a b)^{\#}\left(a b b^{\oplus}\right)^{2} b \\
& =b^{\oplus} b b(a b)^{\#}\left(a b b^{\oplus}\right)^{2} b=b^{\oplus} b\left(a b b^{\oplus}\right)^{\oplus}\left(a b b^{\oplus}\right)^{2} b \\
& =b^{\oplus} b a b b^{\oplus} b=b^{\oplus b a b .}
\end{aligned}
$$

Then, we get

$$
(a b)^{\#}=a b\left((a b)^{\#}\right)^{2}=b^{\oplus} b a b\left((a b)^{\#}\right)^{2}=b^{\oplus} b(a b)^{\#}=b^{\oplus}\left(a b b^{\oplus}\right)^{\oplus} .
$$


Our next aim is to prove $(a b)^{\oplus}=b^{\oplus}\left(a b b^{\oplus}\right)^{\oplus}$. Obviously, $b^{\oplus}\left(a b b^{\oplus}\right)^{\oplus} \in a b\{3\}$. Note that

$$
b^{\oplus}\left(a b b^{\boxplus}\right)^{\oplus}(a b)^{2}=(a b)^{\#}(a b)^{2}=a b
$$

and

$$
a b\left(b^{\oplus}\left(a b b^{\oplus}\right)^{\oplus}\right)^{2}=a b\left((a b)^{\#}\right)^{2}=(a b)^{\#}=b^{\oplus}\left(a b b^{\oplus}\right)^{\oplus},
$$

which imply $a b \in R^{\oplus}$ and $(a b)^{\oplus}=b^{\oplus}\left(a b b^{\oplus}\right)^{\oplus}$ by Lemma 2.1 .

Remark 4.2. Any item of (i)-(iv) in Theorem 4.1 can imply that ab is EP. Indeed, this can be obtained by Lemma 2.7.

Next, characterizations for the equality $a^{\boxplus}=b(a b)^{\#}$ are presented. In particular, we will see that such equivalent conditions also ensure that $a b$ is EP.

Theorem 4.3. Let $a, b \in R^{\oplus}, a b \in R^{\#}$. Then the following are equivalent:

(i) $a^{\boxplus}=b(a b)^{\#}$;

(ii) $a^{\oplus} a b=b a a^{\oplus}$ and $a R \subseteq a b R$;

(iii) $a b \in R^{\boxplus}$ with $(a b)^{\#}=(a b)^{\boxplus}=b^{\oplus} a^{\boxplus}$, and $a R \subseteq b R$;

(iv) $a b b^{\oplus} \in R^{\oplus}$ with $\left(a b b^{\oplus}\right)^{\oplus}=b b^{\oplus} a^{\oplus},(a b)^{\#}=b^{\oplus}\left(a b b^{\oplus}\right)^{\oplus}$, and $a R \subseteq b R$;

(v) $a b b^{\oplus} \in R^{\oplus}$ with $\left(a b b^{\oplus}\right)^{\oplus}=b b^{\oplus} a^{\oplus}=b(a b)^{\#}$, and $a R \subseteq b R$.

Proof. (i) $\Rightarrow$ (ii) Suppose that $a^{\boxplus}=b(a b)^{\#}$, then

$$
a^{\oplus} a b=b(a b)^{\#} a b=b a\left(b(a b)^{\#}\right)=b a a^{\oplus} .
$$

Also, we have $a=a a^{\oplus} a=a b(a b)^{\#} a$, which implies $a R \subseteq a b R$.

(ii) $\Rightarrow$ (iii) There exists $r_{1} \in R$ such that $a=a b r_{1}$, since $a R \subseteq a b R$. Then

$$
a=a^{\oplus} a^{2}=\left(a^{\oplus} a b\right) r_{1} a=b a a^{\oplus} r_{1} a .
$$

Let $r_{2}=a a^{\oplus} r_{1} a$. Then, $a=b r_{2}$, which gives $a R \subseteq b R$.

Since

$$
b^{\oplus}\left(a^{\oplus} a b\right)=b^{\oplus} b a a^{\oplus}=b^{\oplus} b b r_{2} a^{\oplus}=b r_{2} a^{\oplus}=a a^{\oplus}
$$

and

$$
a b b^{\oplus} a^{\oplus}=a b b^{\oplus} a\left(a^{\oplus}\right)^{2}=a b b^{\oplus} b r_{2}\left(a^{\oplus}\right)^{2}=a b r_{2}\left(a^{\oplus}\right)^{2}=a^{2}\left(a^{\oplus}\right)^{2}=a a^{\oplus},
$$

we have $b^{\oplus} a^{\oplus} \in a b\{3,5\}$.

Note that $\left(a b b^{\oplus} a^{\oplus}\right) a b=a a^{\oplus} a b=a b$ and $b^{\oplus} a^{\boxplus}\left(a b b^{\oplus} a^{\oplus}\right)=b^{\oplus} a^{\oplus} a a^{\oplus}=b^{\oplus} a^{\oplus}$, which gives $b^{\oplus} a^{\oplus} \in a b\{1,2\}$. Hence, $a b \in R^{\#}$ with $(a b)^{\#}=b^{\oplus} a^{\oplus}$. In addition, it is easy to see that $(a b)^{\oplus}=b^{\oplus} a^{\oplus}$. In fact,

$$
b^{\boxplus} a^{\oplus}(a b)^{2}=(a b)^{\#}(a b)^{2}=a b \text { and } a b\left(b^{\oplus} a^{\boxplus}\right)^{2}=a b\left((a b)^{\#}\right)^{2}=(a b)^{\#}=b^{\oplus} a^{\boxplus} .
$$


(iii) $\Rightarrow$ (i) Obviously, $b(a b)^{\#} \in a\{2,3\}$. Observe that $a=b r_{3}$ for some $r_{3} \in R$. Then, we have

$$
\begin{aligned}
a & =a\left(a^{\boxplus}\right)^{2} a^{2}=b r_{3}\left(a^{\boxplus}\right)^{2} a^{2}=b b^{\boxplus} b r_{3}\left(a^{\boxplus}\right)^{2} a^{2}=b b^{\oplus} a\left(a^{\oplus}\right)^{2} a^{2} \\
& =b b^{\oplus} a^{\oplus} a^{2}=b(a b)^{\#} a^{2},
\end{aligned}
$$

which implies that $a R \subseteq b(a b)^{\#} R$. Therefore, $a \in R^{\boxplus}$ and $a^{\boxplus}=b(a b)^{\#}$ by Theorem 3.1.

(iii) $\Rightarrow$ (iv) Since $a=b r_{4}$ for some $r_{4} \in R$, we get

$$
b b^{\oplus} a^{\boxplus}=b b^{\oplus} a\left(a^{\oplus}\right)^{2}=b b^{\oplus} b r_{4}\left(a^{\boxplus}\right)^{2}=b r_{4}\left(a^{\oplus}\right)^{2}=a\left(a^{\oplus}\right)^{2}=a^{\oplus} .
$$

Therefore, we only need to prove $a b b^{\oplus} \in R^{\oplus}$ with $\left(a b b^{\oplus}\right)=a^{\oplus}$. From $a\left(b b^{\oplus} a^{\oplus}\right)=a a^{\oplus}$, it follows that $a^{\boxplus} \in a b b^{\oplus}\{3\}$. Also, we have

$$
a b b^{\oplus}\left(a^{\oplus}\right)^{2}=\left(a b b^{\oplus} a^{\oplus}\right) a^{\oplus}=a a^{\oplus} a^{\oplus}=a^{\oplus}
$$

and

$$
a^{\boxplus}\left(a b b^{\oplus}\right)^{2}=a^{\oplus}\left(a b b^{\oplus} a^{\oplus}\right) a^{2} b b^{\oplus}=a^{\oplus} a a^{\oplus} a^{2} b b^{\oplus}=a b b^{\oplus} .
$$

Hence, we have $\left(a b b^{\oplus)}=a^{\oplus}\right.$ by Lemma 2.1. Then, it is easy to see that $(a b)^{\#}=b^{\oplus}\left(a b b^{\oplus}\right)^{\oplus}$.

(iv) $\Rightarrow$ (v) It is obvious.

(v) $\Rightarrow$ (i) From the proof of (iii) $\Rightarrow$ (iv), we know $a^{\oplus}=b b^{\oplus} a^{\oplus}$, which gives $a^{\oplus}=$ $b(a b)^{\#}$.

The next theorem gives the result related to $a^{\oplus}=b(a b)^{\oplus}$.

Theorem 4.4. Let $a \in R^{\#}, a b \in R^{\boxplus}$. Then the following are equivalent:

(i) $a \in R^{\oplus}$ and $a^{\oplus}=b(a b)^{\oplus}$;

(ii) $a R \subseteq b a b R$.

Proof. (i) $\Rightarrow$ (ii) Suppose that $a^{\boxplus}=b(a b)^{\oplus}$. Then, we have

$$
a=a^{\oplus} a^{2}=b(a b)^{\oplus} a^{2}=b a b\left((a b)^{\oplus}\right)^{2} a^{2},
$$

which implies that $a R \subseteq b a b R$.

(ii) $\Rightarrow$ (i) Since $a R \subseteq b a b R$, there exists $w \in R$ such that $a=b a b w$. Then, we obtain

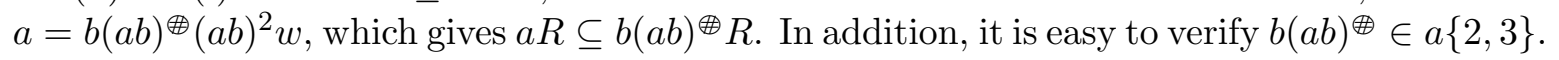
Hence, by Theorem 3.1, it follows that $a \in R^{\oplus}$ with $a^{\oplus}=b(a b)^{\oplus}$.

Finally, we characterize the mixed-type reverse order laws $(a b)^{\#}=b^{\oplus} a^{\oplus}$ and $(b a)^{\#}=$ $a^{\boxplus} b^{\boxplus}$ by the following necessary and sufficient conditions.

Theorem 4.5. Let $a, b \in R^{\oplus}$. Then the following are equivalent:

(i) $a b, b a \in R^{\#}$ with $(a b)^{\#}=b^{\oplus} a^{\oplus},(b a)^{\#}=a^{\oplus} b^{\oplus}$;

(ii) $a^{\oplus} a b=b a a^{\oplus}, b^{\oplus} b a=a b b^{\oplus}$, and $a a^{\oplus} b^{\oplus}=b^{\oplus} a^{\oplus} a$. 
Proof. (i) $\Rightarrow$ (ii) Since $(a b)^{\#}=b^{\oplus} a^{\oplus}$, we have

$$
a b=b^{\oplus} a^{\oplus}(a b)^{2}=b^{\oplus} b\left(b^{\oplus} a^{\oplus}(a b)^{2}\right)=b^{\oplus b a b}
$$

and

$$
a b=(a b)^{2} b^{\oplus} a^{\oplus}=\left((a b)^{2} b^{\oplus} a^{\oplus}\right) a a^{\oplus}=a b a a^{\oplus} .
$$

Similarly, from $(b a)^{\#}=a^{\oplus} b^{\oplus}$, it follows that $b a=a^{\oplus} a b a=b a b b^{\oplus}$. Hence,

$$
a^{\oplus} a b=\left(a^{\oplus} a b a\right) a^{\oplus}=b a a^{\oplus} \text { and } b^{\oplus} b a=\left(b^{\oplus} b a b\right) b^{\oplus}=a b b^{\oplus} .
$$

Since $a b, b a \in R^{\#}$, from Lemma 2.6, it follows that $a(b a)^{\#}=a b\left((a b)^{\#}\right)^{2} a=(a b)^{\#} a$, which immediately yields $a a^{\oplus} b^{\oplus}=b^{\oplus} a^{\oplus} a$.

(ii) $\Rightarrow$ (i) By the hypotheses, we deduce

$$
\begin{aligned}
& a b b^{\oplus}\left(a^{\oplus} a b\right)=a b b^{\oplus b a a^{\oplus}}=a\left(b a a^{\boxplus}\right)=a a^{\oplus} a b=a b, \\
& \left(b^{\oplus} a^{\oplus} a\right) b b^{\oplus} a^{\oplus}=a a^{\oplus} b^{\oplus} b b^{\oplus} a^{\oplus}=\left(a a^{\oplus} b^{\oplus}\right) a^{\oplus}=b^{\oplus} a^{\oplus} a a^{\oplus}=b^{\oplus} a^{\oplus}
\end{aligned}
$$

and

$$
b^{\oplus}\left(a^{\oplus} a b\right)=\left(b^{\oplus} b a\right) a^{\boxplus}=a b b^{\oplus} a^{\oplus},
$$

which imply $b^{\oplus} a^{\oplus} \in a b\{1,2,5\}$, i.e., $(a b)^{\#}=b^{\oplus} a^{\oplus}$. Similarly, we can obtain $(b a)^{\oplus}=$ $a^{\oplus} b^{\oplus}$.

\section{Acknowledgements.}

This research was supported by China Postdoctoral Science Foundation (No. 2018M632385), the National Natural Science Foundation of China (No. 11771076), the Natural Science Foundation of Jiangsu Province (No. BK20141327), the Portuguese Funds through FCT"Fundação para a Ciência e a Tecnologia", within the project UID/MAT/00013/2013.

\section{References}

[1] O.M. Baksalary, G. Trenkler, Core inverse of matrices, Linear Multilinear Algebra 58 (2010) 681-697.

[2] O.M. Baksalary, G. Trenkler, Problem 48-1: reverse order law for the core inverse, Image 48 (2012) 40.

[3] J. Benítez, X.J. Liu, J. Zhong, Some results on matrix partial orderings and reverse order law, Electron. J. Linear Algebra 20 (2010) 254-273.

[4] J.L. Chen, H.H. Zhu, P. Patrício, Y.L. Zhang, Characterizations and representations of core and dual core inverses, Canad. Math. Bull. 60 (2017) 269-282. 
[5] R.E. Cline, An application of representation for the generalized inverse of a matrix, MRC Technical Report 592, 1965.

[6] N. Cohen, E.A. Herman, S. Jayaraman, Solution to problem 48-1: reverse order law for the core inverse, Image 49 (2012) 46-47.

[7] D.S. Cvetković-Ilić, M. Djikić, Various solutions to reverse order law problems, Linear Multilinear Algebra 64 (2016) 1207-1219.

[8] D.S. Cvetković-Ilić, R. Harte, Reverse order laws in $C^{*}$-algebras, Linear Algebra Appl. 434 (2011) 1388-1394.

[9] D.S. Cvetković-Ilić, V. Pavlović, A comment on some recent results concerning the reverse order law for \{1,3,4\}-inverses, Appl. Math. Comput. 217 (2010) 105-109.

[10] C.Y. Deng, Reverse order law for the group inverses, J. Math. Anal. Appl. 382 (2011) 663-671.

[11] N.Č. Dinčić, D.S. Djordjević, Hartwig's triple reverse order law revisited, Linear Multilinear Algebra 62 (2014) 918-924.

[12] M.P. Drazin, Pseudo-inverses in associative rings and semigroups, Amer. Math. Monthly 65 (1958) 506-514.

[13] T.N.E. Greville, Note on the generalized inverse of a matrix product, SIAM Rev. 8 (1966) 518-521.

[14] R.E. Hartwig, Block generalized inverses, Arch, Ration. Mech. Anal. 61 (1976) 197-251.

[15] R.E. Hartwig, J. Luh, A note on the group structure of unit regular ring elements, Pacific J. Math. 71 (1977) 449-461.

[16] S. Izumino, The product of operators with closed range and an extension of the reverse order law, Tohoku Math. J. 34 (1982) 43-52.

[17] X.J. Liu, S.Q. Fu, Y.M. Yu, An invariance property of operator products related to the mixed-type reverse order laws, Linear Multilinear Algebra 64 (2016) 885-896.

[18] X.J. Liu, M. Zhang, J. Benítez, Further results on the reverse order law for the group inverse in rings, Appl. Math. Comput. 229 (2014) 316-326.

[19] X. Mary, Reverse order law for the group inverse in semigroups and rings, Comm. Algebra 43 (2015) 2492-2508.

[20] D. Mosić, N.Č. Dinčić, Reverse order law $(a b)^{\dagger}=b^{\dagger}\left(a^{\dagger} a b b^{\dagger}\right)^{\dagger} a^{\dagger}$ in rings with involution, Filomat 28 (2014) 1791-1815. 
[21] D. Mosić, D.S. Djordjević, Reverse order law for the Moore-Penrose inverse in $C^{*}$ algebras, Electron. J. Linear Algebra 22 (2011) 92-111.

[22] D. Mosić, D.S. Djordjević, Some results on the reverse order law in rings with involution, Aequat. Math. 83 (2012) 271-282.

[23] D. Mosić, D.S. Djordjević, Reverse order law for the group inverse in rings, Appl. Math. Comput. 219 (2012) 2526-2534.

[24] D. Mosić, D.S. Djordjević, The reverse order law $(a b)^{\#}=b^{\dagger}\left(a^{\dagger} a b b^{\dagger}\right)^{\dagger} a^{\dagger}$ in rings with involution, Revista de la Real Academia de Ciencias Exactas, Físicas y Naturales. Serie A. Matemáticas 109 (2015) 257-265.

[25] V. Pavlović, D.S. Cvetković-Ilić, Applications of completions of operator matrices to reverse order law for $\{1\}$-inverses of operators on Hilbert spaces, Linear Algebra Appl. 484 (2015) 219-236.

[26] R. Penrose, A generalized inverse for matrices, Proc. Cambridge Philos. Soc. 51 (1955) 406-413.

[27] P. Puystjens, R.E. Hartwig, The group inverse of a companion matrix, Linear Multilinear Algebra 43 (1997) 137-150.

[28] D.S. Rakić, N.Č. Dinčić, D.S. Djordjević, Group, Moore-Penrose, core and dual core inverse in rings with involution, Linear Algebra Appl. 463 (2014) 115-133.

[29] Y. Tian, On mixed-type reverse-order laws for the Moore-Penrose inverse of a matrix product, Int. J. Math. Math. Sci. 58 (2004) 3103-3116.

[30] H.X. Wang, X.J. Liu, Characterizations of the core inverse and the core ordering, Linear Multilinear Algebra 63 (2015) 1829-1836.

[31] S.Z. Xu, J.L. Chen, X.X. Zhang, New characterizations for core inverses in rings with involution, Front. Math. China 12 (2017) 231-246. 